\title{
Sanguinarine inhibits invasiveness and the MMP-9 and COX-2 expression in TPA-induced breast cancer cells by inducing HO-1 expression
}

\author{
SUN YOUNG PARK ${ }^{1}$, MEI LING JIN ${ }^{2}$, YOUNG HUN KIM ${ }^{1}$, SANG-JOON LEE ${ }^{2}$ and GEUNTAE PARK ${ }^{3}$ \\ ${ }^{1}$ Bio-IT Fusion Technology Research Institute, ${ }^{2}$ Department of Microbiology, ${ }^{3}$ Institute for Research and \\ Industry Cooperation, Pusan National University, Busan 609-735, Republic of Korea
}

Received September 23, 2013; Accepted October 29, 2013

DOI: $10.3892 /$ or.2013.2843

\begin{abstract}
Most complications of breast cancer are attributed to metastasis to distant organs, including lymph nodes, bone, lung and liver. Metastasis is considered the leading cause of mortality in patients with breast cancer. The emergence of antimetastatic properties in breast cancer is an important clinical phenomenon affecting long-term survival. In the present study, we investigated the anti-invasive mechanism of sanguinarine by focusing on its role in inducing HO-1 in breast cancer cells. The results showed that sanguinarine inhibited TPA-induced MMP-9 and COX-2 mRNA and protein expression in a dose-dependent manner at non-cytotoxic concentrations. Similarly, the MMP-9 enzymatic activity and the PGE $_{2}$ levels significantly decreased in MCF-7 breast cancer cells. TIMP-1 and TIMP-2, specific endogenous inhibitors of MMP-9, were slightly induced by sanguinarine. Subsequent studies revealed that sanguinarine suppressed TPA-induced NF- $\kappa \mathrm{B}$ and AP-1 activation, as well as the phosphorylation of Akt and ERK. Furthermore, sanguinarine significantly inhibited TPA-induced invasion and migration in breast cancer cells We also demonstrated that sanguinarine induced HO-1 expression, and that the inhibition of MMP-9 and COX-2 expression and the enzymatic activity of sanguinarine were abrogated by siRNA-mediated knockdown of HO-1 expression. Thus, knockdown of endogenous HO-1 decreased TPA-induced cell invasion. Overall, the results of the present study demonstrate that HO-1 plays a pivotal role in the anti-invasive response of sanguinarine in TPA-stimulated breast cancer cells.
\end{abstract}

\section{Introduction}

Breast cancer is one of the leading causes of cancer-related mortality among females in the developed world. Although

Correspondence to: Professor Geuntae Park, Institute for Research and Industry Cooperation, Pusan National University, 30 JangjeonDong, Busan 609-735, Republic of Korea

E-mail: gtpark@pusan.ac.kr

Key words: sanguinarine, invasion, TPA, MMP-9, COX-2, HO-1 therapies for human breast cancer have been developed, the mortality rate of breast cancer patients has remained unchanged. Therefore, the development of new strategies for more effective treatment is highly desirable, and chemotherapeutic use of phytochemicals as anticancer agents has received recent attention due to their low cytotoxicities and the low cost of plant-derived raw materials $(1,2)$.

Since malignancy of tumors is generally attributed to their invasive and metastatic capacity, a therapeutic agent that only possesses the ability to induce apoptosis might not be effective against metastatic tumors. Metastasis is a multi-step process that involves the detachment of cancer cells from the primary tumor as well as their migration, adhesion and invasion into lymphatic vessels or blood. Next, extravasation from the vessels is mediated by the action of extracellular proteases, among which the matrix metalloproteinases (MMPs) have been demonstrated to play crucial roles. In particular, type IV collagenases or gelatinases (e.g., MMP-2 and MMP-9) are major enzymes in the degradation of a variety of extracellular matrix (ECM) components, leading to tumor migration, invasion and metastasis in numerous types of cancer $(3,4)$. COX-2 is overexpressed throughout breast cancer progression. The upregulation of COX-2 and $\mathrm{PGE}_{2}$ might be involved in cancer cell invasion by stimulating the expression of MMPs. In fact, both MMP-2 and MMP-9 are overexpressed in breast cancer and are closely associated with metastasis, poor prognosis and a high mortality rate in breast cancer patients. Therefore, inhibition of MMP activity and expression is important for blocking the metastatic ability of breast cancer cells (3).

In general, MMP-2 is constitutively overexpressed in highly metastatic tumors, whereas MMP-9 can be stimulated by the inflammatory cytokine tumor necrosis factor (TNF)- $\alpha$, the epidermal growth factor and phorbol esters through the activation of distinct intracellular signaling pathways. Moreover, stimulators, including cytokines and 12- $O$-tetradecanoylphorbol-13-acetate (TPA), regulate the expression of MMP-9 and COX-2 by controlling the activation of transcription factors, such as the nuclear factor (NF)- $\kappa \mathrm{B}$ and the activator protein (AP)-1, since the promoter regions of MMP-9 and COX-2 possess NF- $\mathrm{BB}$-binding sites $(5,6)$. Two transcription factors, NF- $\kappa \mathrm{B}$ and $\mathrm{AP}-1$, regulate the expression of a number of genes and of the products associated with 
inflammation, tumorigenesis and metastasis. In fact, NF- $\kappa \mathrm{B}$ and AP-1 are major transcription factors involved in the activation of genes encoding inflammatory cytokines, such as IL-1 $\beta$ and TNF- $\alpha$. Additionally, NF- $\kappa \mathrm{B}$ can induce the activation of COX-2 and MMP-9. Several reports have indicated that the early suppression of MMP-9 and COX-2 enzyme activity or expression could be used for preventing invasion and cancer metastasis (6-8). Therefore, agents possessing the ability to inhibit the expression of MMP-9 or COX-2 warrant investigation with regards to treatment of cancer cell invasion and metastasis.

HO-1 is an inducible enzyme that catalyzes the ratelimiting step for the oxidative degradation of cellular heme into carbon monoxide, biliverdin and free iron (9). HO-1 and its enzymatic byproducts provide host defense mechanisms such as antioxidant and anti-inflammatory effects. Experimental evidence has established HO-1 as a critical component of multiple signaling pathways that regulate proliferation and metastasis. Increased HO-1 expression likely plays an important role in the development and progression of breast cancer $(10,11)$. Therefore, HO-1 might be an important therapeutic target for the treatment of human breast cancer.

In traditional Chinese medicine, Macleaya cordata (plume poppy) has long been used as a painkiller and an anti-inflammatory agent in humans. Macleaya cordata is a plant of the Papaveraceae family, which includes abundant bioactive compounds, mostly isoquinoline alkaloids such as allocryptopine, angoline, berberine, bocconine, bocconoline, chelerythrine, heleritrine, macarpine, protopine and sanguinarine $(12,13)$. The capsule of $M$. cordata contains the highest level of sanguinarine and chelerythrine. Moreover, the highest amount of protopine and allocryptopine was found in the footstalks. In addition, $M$. cordata is recorded in the European Food Safety Authority (EFSA) list of plants utilized as ingredients of feed additives in animal production due to their anti-inflammatory activity. Sanguinarine and chelerythrine are biologically active components of these extracts (13-15). The chemical name of sanguinarine is 13-methyl[1,3] benzodioxole[5,6-c]-1,3-dioxolan[4,5-i] phenanthridinium. In particular, sanguinarine is noted for its pharmacological activity, e.g., its antihypertensive, cardiac and antitumor properties $(14,16,17)$. In the present study, we investigated the inhibitory activity of sanguinarine on the TPA-induced upregulation of MMP-9 and COX-2 in human MCF-7 breast cancer cells. Here, we provide evidence that sanguinarine inhibits TPA-induced MMP-9 and COX-2 expression by blocking NF- $\kappa \mathrm{B}, \mathrm{Akt}$ and ERK1/2 signaling. Furthermore, we showed that sanguinarine inhibits the migration and invasion of breast cancer cells. Sanguinarine exhibits an anti-invasive activity related to the induction of HO-1 expression. These findings provide new insights into the mechanism by which sanguinarine mediates its anti-invasive activity and might thus be useful for developing novel therapeutic strategies to target breast cancer metastasis.

\section{Materials and methods}

Materials and reagents. Sanguinarine and other chemicals were purchased from Sigma-Aldrich (St. Louis, MO, USA). BioCoat $^{\mathrm{TM}}$ Matrigel $^{\mathrm{TM}}$ invasion chambers were obtained from
BD Biosciences (San Jose, CA, USA). Antibodies against phosphorylated p38 (p-p38), p-JNK, p-ERK, p-IкB- $\alpha$, MMP-2 and MMP-9 were purchased from Cell Signaling Technology (Beverly, MA, USA). HO-1 small interfering RNA (siRNA) and antibodies against COX-1, COX-2, ERK, JNK, p38, c-Jun, $\mathrm{c}-\mathrm{Fos}, \mathrm{NF}-\kappa \mathrm{B}$ and TBP were purchased from Santa Cruz Biotechnology (Santa Cruz, CA, USA). Cell culture medium RPMI-1640 and fetal bovine serum (FBS) were purchased from Invitrogen Life Technologies (Carlsbad, CA, USA). The FuGENE-6 transfection reagent and the X-tremeGENE siRNA transfection reagent were purchased from Roche Diagnostics (Indianapolis, IN, USA).

Cell cultures. Human breast cancer cell line MCF-7 was obtained from the American Type Culture Collection (ATCC, Manassas, VA, USA). Cells were grown in RPMI supplemented with $10 \%$ heat-inactivated FBS and $1 \%$ penicillin-streptomycin at $37^{\circ} \mathrm{C}$ in a humidified incubator in a $5 \% \mathrm{CO}_{2}$ atmosphere.

Cell invasion assay. The cell invasion assay was conducted using BioCoat ${ }^{\mathrm{TM}}$ Matrigel $^{\mathrm{TM}}$ invasion chambers according to the manufacturer's instructions. Briefly, the Matrigel coating was rehydrated in $0.5 \mathrm{ml}$ of Dulbecco's modified Eagle's medium (DMEM) for $30 \mathrm{~min}$ immediately before the experiments. Cells $\left(5 \times 10^{4}\right)$ suspended in $0.5 \mathrm{ml}$ of serum-free medium were added to the upper chamber of the Matrigel-coated filter inserts. Following treatment with sanguinarine for $1 \mathrm{~h}, 0.5 \mathrm{ml}$ of serum-free medium containing $50 \mathrm{nM}$ TPA was added to the bottom well as a chemoattractant. The chambers were then incubated for $24 \mathrm{~h}$. Following incubation, cells on the upper side of the chamber were removed using cotton swabs, and cells that had migrated were fixed and stained with $2 \%$ ethanol containing $0.2 \%$ crystal violet powder. Invading cells were enumerated under a light microscope at x10 magnification.

In vitro wound-healing repair assay. For the in vitro wound-healing repair assay (cell migration assay), the cells were seeded in a 24-well culture dish until they reached $90 \%$ confluence. The cells were then maintained in serum-free medium for $12 \mathrm{~h}$. The monolayers were carefully scratched using a $200-\mu 1$ pipette tip. Cellular debris was removed by washing with phosphate-buffered saline (PBS), and the cells were incubated in serum-free medium. The migrating cells were then fixed with cold $75 \%$ methanol for $30 \mathrm{~min}$ and washed three times with PBS. The cultures were photographed at 0 and $24 \mathrm{~h}$ to monitor the migration of cells into the wounded area, and then the closure of the wounded area was calculated.

Gelatin zymography assay. The enzyme activities of MMP-2 and MMP-9 in conditioned medium were determined using the gelatin zymography protease assay. Briefly, cells $\left(2 \times 10^{5}\right)$ were seeded in 6-well plates and allowed to grow at $80 \%$ confluence. The cells were then maintained in serum-free medium for $12 \mathrm{~h}$ prior to treatment with sanguinarine and TPA for $24 \mathrm{~h}$. Conditioned media were collected, cleared by centrifugation, and mixed with $2 X$ SDS sample buffer (Invitrogen Life Technologies), followed by electrophoresis in a polyacrylamide gel containing $0.1 \%(\mathrm{w} / \mathrm{v})$ gelatin. Following electrophoresis, the gels were incubated in renaturing buffer (2.5\% Triton $\mathrm{X}-100)$ with gentle agitation to remove SDS, 
followed by incubation in developing buffer $(50 \mathrm{mM}$ Tris- $\mathrm{HCl}$, $\mathrm{pH} 7.4$ and $10 \mathrm{mM} \mathrm{CaCl}$ ) overnight at $37^{\circ} \mathrm{C}$ to allow digestion of the gelatin. Gels were then stained with SimplyBlue ${ }^{\mathrm{TM}}$ SafeStain (Invitrogen Life Technologies) until clear bands suggestive of gelatin digestion appeared.

Western blot analysis. Cells were harvested in ice-cold lysis buffer consisting of $1 \%$ Triton X-100, $1 \%$ deoxycholate and $0.1 \%$ SDS. The protein content of the cell lysates was then determined using the Bradford reagent (Bio-Rad Laboratories, Hercules, CA, USA). Proteins in each sample (50 $\mu \mathrm{g}$ of total protein) were resolved by $12 \%$ SDS-PAGE, transferred to a polyvinylidene difluoride membrane, and exposed to the appropriate antibodies. The proteins were visualized with the Enhanced Chemiluminescence Detection system (Amersham Biosciences, Piscataway, NJ, USA) using horseradish peroxidase (HRP)-conjugated anti-rabbit or anti-mouse secondary antibodies. Images were acquired using an ImageQuant 350 analyzer (Amersham Biosciences).

Real-time PCR. Total cellular RNA was isolated using an RNAspin Mini Isolation kit (GE Healthcare, Buckinghamshire, UK) according to the manufacturer's instructions. One microgram of total RNA was reverse-transcribed using Maxime RT PreMix (Intron Biotechnology, Seongnam, Korea) and anchored oligo-dT ${ }_{15}$ primers. Real-time PCR was performed with SYBR ${ }^{\circledR}$-Green Master Mix (Applied Biosystems, Foster City, CA, USA) using a Chromo4 instrument (Bio-Rad Laboratories). The relative amount of target mRNA was determined using the $\mathrm{Ct}$ method by normalizing target mRNA $\mathrm{Ct}$ values to those for GAPDH $(\Delta \mathrm{Ct})$. The real-time PCR cycling conditions were: $95^{\circ} \mathrm{C}$ for $5 \mathrm{~min}, 95^{\circ} \mathrm{C}$ for $30 \mathrm{sec}$ for 40 cycles, $55^{\circ} \mathrm{C}$ for $20 \mathrm{sec}$ and $72^{\circ} \mathrm{C}$ for $30 \mathrm{sec}$, followed by fluorescence measurement. The primer sequences used were as follows: MMP-9-sense (5'-TTCCCTGGAGACCTGAGAACC-3'), MMP-9-antisense (5'-CGGCAAGTCTTCCGAGTAGTTT-3'), COX-2-sense (5'-TACAAGCAGTGGCAAAGGC-3'), COX-2antisense (5'-AGATCATCTCTGCCTGAGTATCTT-3'), GAPDH-sense (5'-AGGTGGTCTCCTCTGACTTC-3') and GAPDH-antisense (5'-TACCAGGAAATGAGCTTGAC-3').

Measurement of prostaglandin $E_{2}$ concentrations. Cells were incubated with different concentrations of sanguinarine for $1 \mathrm{~h}$ and then with TPA for $23 \mathrm{~h}$. The prostaglandin $\mathrm{E}_{2}$ levels were quantified in the culture medium using an enzyme-linked immunosorbent assay (ELISA) kit (Cayman Chemical, Ann Arbor, MI, USA) according to the manufacturer's instructions.

Chromatin immunoprecipitation (ChIP) assay. To detect the in vivo association of nuclear proteins with the human MMP-9 promoter, ChIP analysis was conducted as previously described (18), with some modifications. Briefly, $2 \times 10^{7}$ cells were incubated in culture medium containing $1 \%$ formaldehyde for $10 \mathrm{~min}$ at room temperature, and the cross-linking reaction was quenched by adding glycine to a final concentration of $0.125 \mathrm{M}$. Isolated nuclei were digested with 200 units of MNase at $37^{\circ} \mathrm{C}$ for $15 \mathrm{~min}$, followed by sonication to produce chromatin of primarily mononucleosomal size. Fragmented chromatin was incubated with antibodies for $3 \mathrm{~h}$ at $4^{\circ} \mathrm{C}$. Protein-DNA complexes were recovered using protein A agarose beads, washed, and then eluted with elution buffer. Cross-links were reversed at $65^{\circ} \mathrm{C}$ in $0.25 \mathrm{M} \mathrm{NaCl}$ overnight, and DNA was digested with proteinase $\mathrm{K}$ for $2 \mathrm{~h}$ at $50^{\circ} \mathrm{C}$. DNA was isolated using a DNA Purification kit (Qiagen). Immunoprecipitated DNA was used for each PCR. PCR primers for the MMP-9 promoter (373 bp including the NF- $\kappa$ B cluster; GenBank accession no. AF538844) were as follows: sense (5'-CACTTCAAAGTGGTAAGA-3'), antisense (5'-GAAAGTGATGGAAGACTCC-3') and for the $\mathrm{COX}-2$ promoter (420 bp including $\mathrm{NF}-\kappa \mathrm{B}$ cluster): sense (5'-TCCCGACGTGACTTCCTCGA-3') and antisense (5'-GGAGAG GAGGGAAAAATTTG-3').

Transient transfection and dual luciferase assay. To determine the promoter activity, we used a Dual-Luciferase Reporter Assay system (Promega Corp., Madison, WI, USA). Cells were transfected with the NF- $\kappa \mathrm{B}$ luciferase reporter plasmid or the AP-1 luciferase reporter plasmid (Agilent Technologies, Santa Clara, CA, USA) using the FuGENE-6 reagent according to the manufacturer's instructions. The Renilla luciferase control plasmid pRL-CMV (Promega Corp.) was co-transfected as an internal control to determine the transfection efficiency. Twenty-four hours following transfection, cells were incubated with the indicated reagents for $1 \mathrm{~h}$ and then treated with TPA for $24 \mathrm{~h}$. The luciferase activity was assayed with a Dual-Luciferase Assay kit (Promega Corp.) according to the manufacturer's instructions. The luminescence was measured with a GloMax ${ }^{\circledR} 96$ Microplate Luminometer (Promega Corp.).

Transient transfection of siRNA. Transfection of MCF-7 cells with siRNA was performed using the X-tremeGENE siRNA transfection reagent, according to the manufacturer's instructions. Commercially available human HO-1-specific and negative control siRNAs were used for transfection. Briefly, $\mathrm{X}$-tremeGENE siRNA transfection reagent $(10 \mu \mathrm{l})$ was added to $100 \mu \mathrm{l}$ of serum-free medium containing $2 \mu \mathrm{g}$ of each siRNA oligo, and the mixture was incubated for $20 \mathrm{~min}$ at room temperature.

Statistical analysis. Each experiment was repeated at least three times and all results are expressed as the mean \pm SE. Statistical analysis was performed using the SPSS software (version 18.0) to determine significant differences. We used one-way analysis of variance (ANOVA) followed by Tukey's post-hoc test for comparisons between three or more groups. Data with $\mathrm{p}<0.05$ were considered to indicate statistically significant differences.

\section{Results}

Sanguinarine inhibits the activity and expression of MMP-9 and COX-2 in human breast cancer cells. MMP-9 and COX-2 have important roles in migration and invasion (5). To explore the effect of sanguinarine on the activity and expression of MMP-9 and COX-2, cells were exposed to different doses of a range of non-toxic concentrations of sanguinarine. First, we performed gelatin zymography and ELISA to assess the activity of MMP-9 and COX-2 in cells exposed to sanguinarine. As shown in Fig. $1 \mathrm{~A}$ and $\mathrm{B}$, sanguinarine significantly 

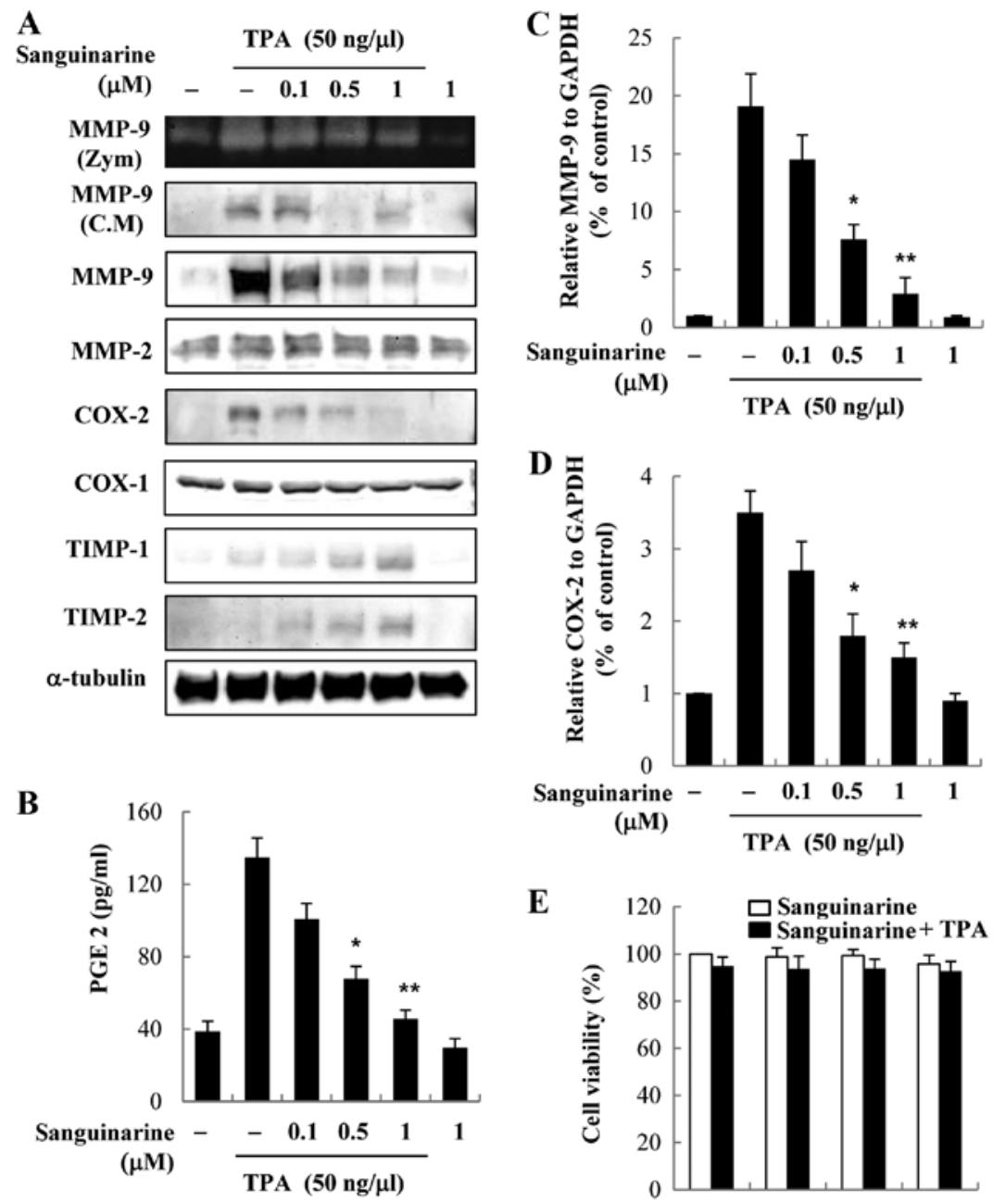

E

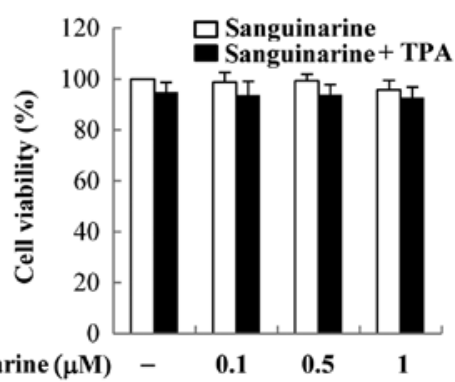

Figure 1. Effects of sanguinarine on matrix metalloproteinase (MMP)-9, MMP-2, COX-1, COX-2, TIMP-1 and TIMP-2 expression in human breast cancer cells. (A) MCF-7 cells were treated with sanguinarine for $1 \mathrm{~h}$, followed by $12-O$-tetradecanoylphorbol-13-acetate (TPA) treatment $(50 \mathrm{ng} / \mathrm{ml})$ for $24 \mathrm{~h}$. The MMP-9 enzymatic activity was analyzed by gelatin zymography (Zym), secretion by western blotting, and intracellular protein expression by western blotting. The protein levels of MMP-9, MMP-2, COX-1, COX-2, TIMP-1 and TIMP-2 were evaluated by western blotting. C.M, conditioned medium. (B) PGE 2 was measured in the culture supernatant using an enzyme-linked immunosorbent assay (ELISA) kit. (C) MMP-9 and (D) COX-2 relative mRNA expression ( $2^{-\triangle C t}$ ) was determined by real-time RT-PCR relative to GAPDH mRNA (by subtracting the $\mathrm{Ct}$ value for GAPDH from the Ct value for MMP-9 and COX-2, i.e.,

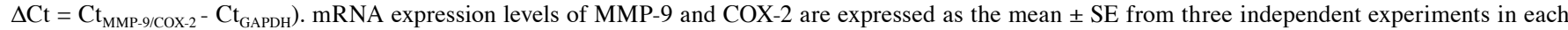
group. "P<0.05; ${ }^{* *} \mathrm{p}<0.01$ (both vs. the TPA-treated group). (F) Effect of sanguinarine on cell viability. Cells were treated with the indicated concentration of sanguinarine in the presence of TPA $(50 \mathrm{ng} / \mathrm{ml})$ for $24 \mathrm{~h}$. Cell viability was determined by the MTT assay. Each bar represents the mean \pm SE from three independent experiments in each group. ${ }^{*} \mathrm{P}<0.05 ;{ }^{* *} \mathrm{p}<0.01$ (both vs. the TPA-treated group).

suppressed the TPA-induced MMP-9 enzymatic activity and the $\mathrm{PGE}_{2}$ production in a dose-dependent manner in MCF-7 breast cancer cells. We further explored the inhibitory activity of sanguinarine on TPA-induced MMP-9 and COX-2 expression at the mRNA and protein levels using western blotting and real-time PCR (Fig. 1A, C and D). The results indicated that the inhibitory effect of sanguinarine on MMP-9 and COX-2 expression and activity was not due to a change in cell viability (Fig. 1E). These results suggested that the activity and expression of MMP-9 and COX-2 were inhibited by sanguinarine in TPA-induced human breast cancer cells.

Sanguinarine stimulates TIMP-1 and TIMP-2. Since the physiological activity of MMP-9 is highly related to that of its specific endogenous inhibitors TIMP-1 and TIMP-2 $(3,4,19)$, western blotting was performed to explore the potential effects of sanguinarine on TIMP-1 and TIMP-2 expression. As shown in Fig. 1A, sanguinarine increased TIMP-1 and TIMP-2 protein expression in a dose-dependent manner in the presence of TPA. In summary, these data indicate the involvement of TIMP-1 and TIMP-2 in the sanguinarine-induced anti-invasive effect in TPA-stimulated human breast cancer cells.

Sanguinarine suppresses MMP-9 and COX-2 expression via the $N F-\kappa B$ and $A P-1$ signaling pathways. MMP-9 and COX-2 expression is regulated by the transcription factors $\mathrm{NF}-\kappa \mathrm{B}$ and AP-1. It has been reported that the binding sites of these transcription factors lie within the MMP-9 and COX-2 promoters $(6,7)$. Therefore, we investigated whether the inhibitory effect of sanguinarine on MMP-9 and COX-2 expression is mediated by the NF- $\kappa \mathrm{B}$ and AP-1 signaling pathways. As shown in Fig. 2A, 30 min of TPA stimulation led to the phosphorylation and nuclear translocation of the NF- $\mathrm{NB}$ subunit p65 in the nuclear extract of MCF-7 cells, and following 


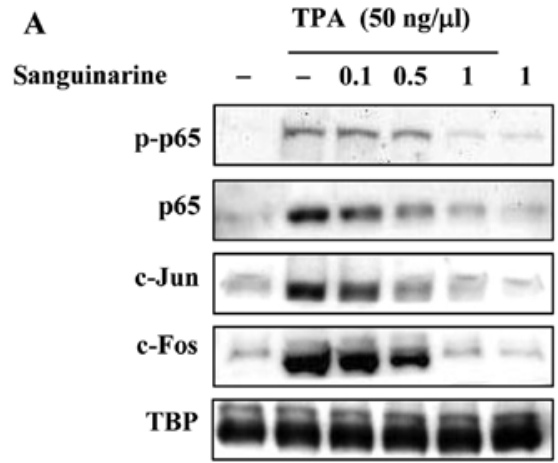

C

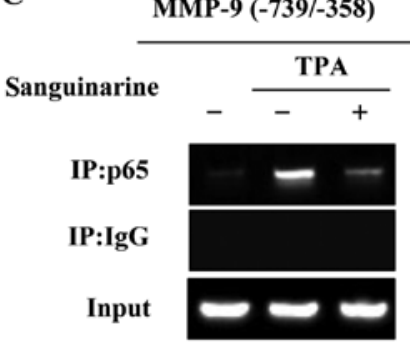

B

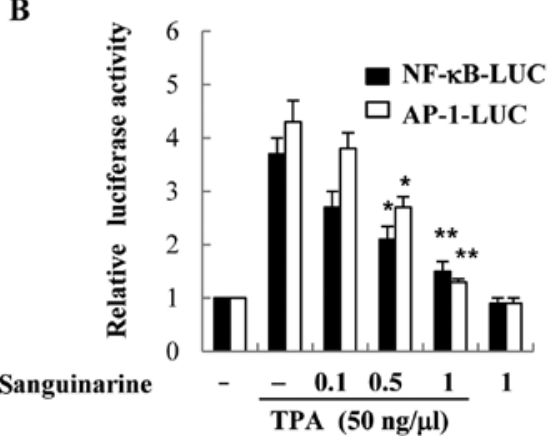

D

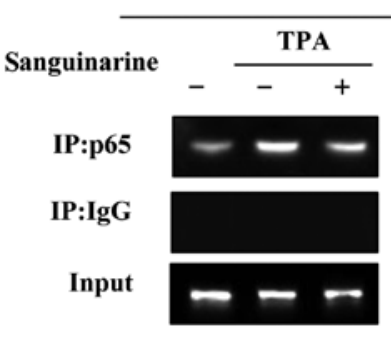

Figure 2. Nuclear factor (NF)- $\mathrm{kB}$ and activator protein (AP)-1 are involved in sanguinarine-mediated downregulation of MMP-9 and COX-2 expression. (A) MCF-7 cells were treated with various concentrations of sanguinarine for $1 \mathrm{~h}$, followed by 12 - $O$-tetradecanoylphorbol-13-acetate (TPA) treatment (50 ng/ml) for $1 \mathrm{~h}$. Nuclear extracts were prepared and analyzed by western blotting using anti-NF-kB p65, -c-Jun and -c-Fos antibodies. (B) Cells were transfected with an $\mathrm{NF}-\mathrm{kB}$ or AP-luciferase reporter plasmid. After $24 \mathrm{~h}$, the cells were incubated with the indicated concentrations of sanguinarine for $1 \mathrm{~h}$, followed by stimulation with TPA $(50 \mathrm{ng} / \mathrm{ml})$ for $24 \mathrm{~h}$. Equal amounts of cell extracts were assayed for the dual-luciferase activity. Expression of the Renilla luciferase control was used to normalize the NF- $\mathrm{kB}$ and the AP-1-luciferase activity. Each bar represents the mean $\pm \mathrm{SE}$ from three independent experiments in each group. ${ }^{*} \mathrm{P}<0.05 ;{ }^{* *} \mathrm{p}<0.01$ (both vs. the TPA-treated group). (C and D) Cells were incubated with sanguinarine for $1 \mathrm{~h}$ and then with TPA for $4 \mathrm{~h}$. DNA immunoprecipitated with the antiNF-kB p65 antibody was purified as described in Materials and methods. The precipitated matrix metalloproteinase (MMP)-9 promoter region (-739 to -358) and the COX-2 promoter region (-550 to -130) were amplified by PCR. The input represents PCR products from chromatin pellets prior to immunoprecipitation (IP).

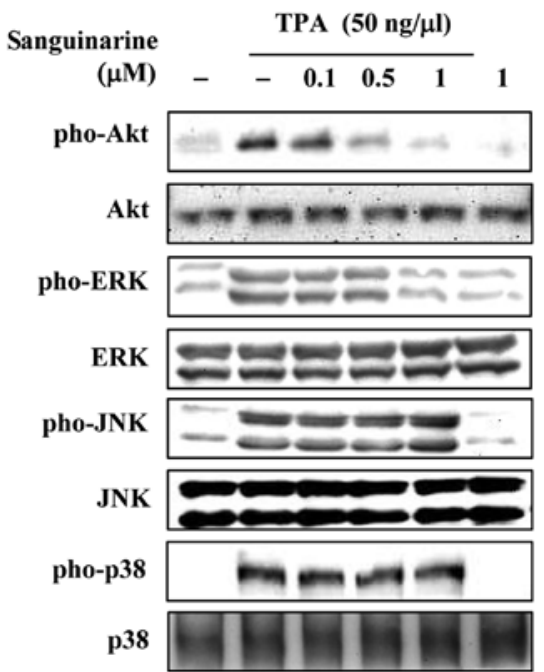

Figure 3. Effect of sanguinarine on 12- $O$-tetradecanoylphorbol-13-acetate (TPA)-induced activation of the Akt and MAPK signaling pathways. MCF-7 cells were treated with the indicated concentrations of sanguinarine for $1 \mathrm{~h}$, followed by stimulation with TPA $(50 \mathrm{ng} / \mathrm{ml})$ for $1 \mathrm{~h}$. Equal amounts of cell extract were analyzed by western blotting with anti-phospho-Akt, -ERK1/2, $-\mathrm{JNK}$ and -p38 antibodies.

treatment with sanguinarine, the phosphorylation and nuclear translocation of p65 were effectively reduced in a dosedependent manner. Similarly, the nuclear translocation level of AP-1 subunits c-Jun and c-Fos was significantly attenuated by sanguinarine in comparison to the TPA-induced cells. To further confirm the effect of sanguinarine on the transactivity of NF- $\kappa \mathrm{B}$ and AP-1, we determined the activity of a luciferase reporter gene containing the $\mathrm{NF}-\kappa \mathrm{B}$ and $\mathrm{AP}-1$ binding regions. As shown in Fig. 2B, treatment of the TPA-stimulated MCF-7 cells with sanguinarine increased the promoter activity of NF- $\kappa \mathrm{B}$ by 3.7-fold and the promoter activity of AP-1 by 4.2-fold; treatment with $1 \mu \mu \mathrm{M}$ sanguinarine decreased the TPA-stimulated promoter activity of NF- $\kappa \mathrm{B}$ by 1.6 -fold and that of AP-1 by 1.4-fold. Next, the binding activity of NF- $\kappa \mathrm{B}$ to the MMP-9 and COX-2 promoters was investigated using a ChIP-PCR assay. A low level of $\mathrm{NF}-\kappa \mathrm{B}$ binding activity to the MMP-9 and COX-2 gene promoters was observed in the unstimulated cells, whereas an important fraction of the $\mathrm{NF}-\kappa \mathrm{B}$ binding activity was induced by TPA treatment. The increased NF- $\kappa \mathrm{B}$ binding activity was dramatically inhibited by sanguinarine (Fig. 2C and D). Overall, these results show that sanguinarine suppresses MMP-9 and COX-2 expression through the suppression of NF- $\mathrm{B}$ and AP-1 activities.

Sanguinarine inhibits Akt and ERK activation in TPA-stimulated breast cancer cells. Although sanguinarine has been shown to inhibit MMP-9 and COX-2 in TPA-stimulated breast cancer cells, the underlying mechanisms are poorly understood. Therefore, we examined the effects of sanguinarine on TPA-induced MAPK phosphorylation using western blot analysis. As shown in Fig. 3, sanguinarine inhibited 
A

\section{Con}

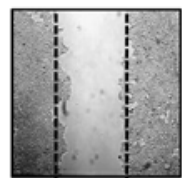

B
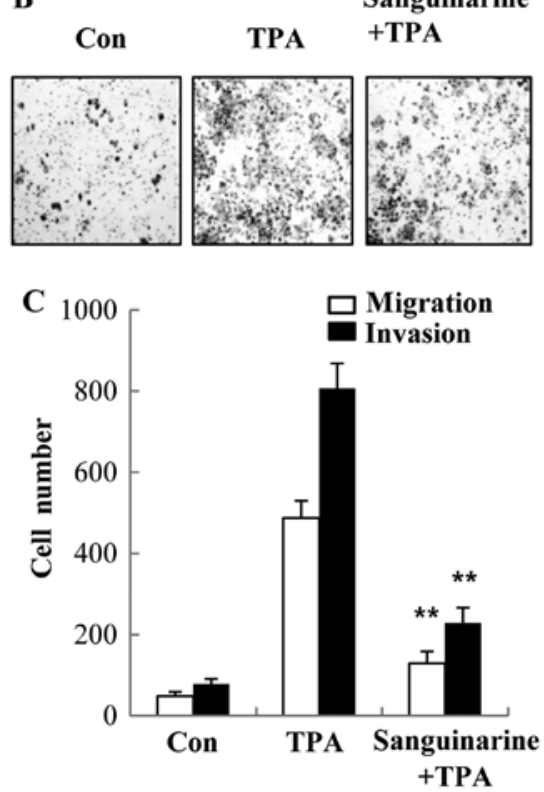

Figure 4. Inhibitory effects of sanguinarine on the migration and invasion of human breast cancer cells. (A) MCF-7 cells were scratched with a pipette tip, pretreated with sanguinarine $(1 \mu \mathrm{M})$, and then treated with 12- $O$-tetradecanoylphorbol-13-acetate (TPA; $50 \mathrm{ng} / \mathrm{ml}$ ) for $24 \mathrm{~h}$. Migrating cells were analyzed by phase contrast microscopy. (B) Cells were treated with sanguinarine $(1 \mu \mathrm{M})$, followed by TPA treatment $(50 \mathrm{ng} / \mathrm{ml})$ for $24 \mathrm{~h}$. Cells that invaded the bottom side of the filter were stained with crystal violet and counted under a microscope. (C) Quantified levels of cell migration and invasion, expressed relative to the basal invasion of TPA-untreated cells. Each bar represents the mean \pm SE from three independent experiments in each group. ${ }^{* *} \mathrm{P}<0.01$ vs. the TPA-treated group.

TPA-induced Akt and ERK activation. However, treatment with up to $1 \mu \mathrm{M}$ sanguinarine had no effect on TPA-induced JNK and p38 phosphorylation. Importantly, treatment with sanguinarine or TPA did not affect the total levels of Akt or ERK. These results indicate that sanguinarine inhibited TPA-induced MMP-9 and COX-2, most likely by inhibiting Akt and ERK activation in breast cancer cells.

Sanguinarine inhibits TPA-induced migration and invasion in human breast cancer cells. Metastasis is a complex and multi-step process and is considered the leading cause of cancer-related mortality (20). Since proteolytic digestion of ECM and migration of cancer cells across the blood vessel-lining endothelial monolayers play crucial roles in the metastatic process, we investigated the effects of sanguinarine on the invasive property of breast cancer cells. The inhibitory effect of sanguinarine on migration of breast cancer cells was determined by a wound-healing assay. The confluent ( $\sim 90 \%$ of the maximum cell density) monolayer was first scraped and then scratched using a micropipette. Following incubation with sanguinarine, cells were stimulated with TPA. The data presented in Fig. 4A indicate that sanguina- rine suppressed TPA-induced cell migration to the denuded area. To further determine the effect of sanguinarine on the invasive activity of human breast cancer cells, the Matrigel Transwell invasion assay was used. The results showed that the number of cells invading the lower chamber was dramatically reduced following sanguinarine treatment when compared to the TPA-treated group in MCF-7 cells (Fig. 4B). Thus, we demonstrate that sanguinarine might be an effective agent in preventing cell migration and invasion of MCF-7 cells.

HO-1 knockdown abolishes the inhibitory effect of sanguinarine on TPA-induced MMP-9 and COX-2 expression and activity. We investigated whether sanguinarine suppresses TPA-induced MMP-9 and COX-2 expression in breast cancer cells through HO-1 expression. First, we examined the mRNA and protein expression of $\mathrm{HO}-1$ in the presence of sanguinarine in MCF-7 breast cancer cells. As shown in Fig. 5A and B, the expression of HO-1 mRNA and protein increased with sanguinarine in a concentration-dependent manner in the presence of TPA, and peaked at $8 \mathrm{~h}$. To investigate the effects of HO-1 silencing, breast cancer cells were transiently transfected with HO-1-siRNA, and the effects of sanguinarine on TPA-induced MMP-9 and COX-2 expression and activity were evaluated. As shown in Fig. 5E and F, HO-1 silencing abrogated HO-1 expression. HO-1 silencing blocked sanguinarine-mediated suppression of TPA-induced MMP-9 and COX-2 expression and activity compared to control siRNA. Sanguinarine-mediated inhibition of invasive activity was also suppressed by knockdown of endogenous HO-1 in breast cancer cells (Fig. 5G). In summary, these results indicate that sanguinarine-dependent HO-1 expression plays a crucial role in downregulation of MMP-9 and COX-2 expression and activation.

\section{Discussion}

Recently, considerable attention has been given to the use of natural products as novel effective anticancer agents in humans $(1,2)$. Sanguinarine is a benzophenanthridine alkaloid with a wide range of preclinical antitumor activities in hepatocellular carcinoma, prostate cancer, osteosarcoma cancer and bladder cancer $(13,15,16)$. Although sanguinarine has been shown to exert antitumor effects in various models, only a few studies have reported the antitumor properties of sanguinarine and the mechanism by which sanguinarine interacts with human breast cancer cells. Therefore, in the present study, we examined the potential role of sanguinarine as a chemotherapeutic agent, in particular during metastasis, using MCF-7 human breast cancer cells.

Metastatic spread of cancer is accountable for $\sim 90 \%$ of cancer-related mortality in humans and remains a serious obstruction to cancer treatment. Thus, inhibiting the metastatic ability of cancer cells has become an important aspect in the development of successful anticancer agents $(3,20)$. In the present study, we aimed to explore the inhibitory effect of sanguinarine on cell migration and invasion in TPA-induced human breast cancer cells. TPA is a well-known tumor promoter that activates most of the protein kinase $\mathrm{C}$ (PKC) isozymes by direct binding. This activation results in a dramatic PKC-mediated induction of tumor cell invasion (5). When human breast cancer cells were treated with sanguinarine at 
A

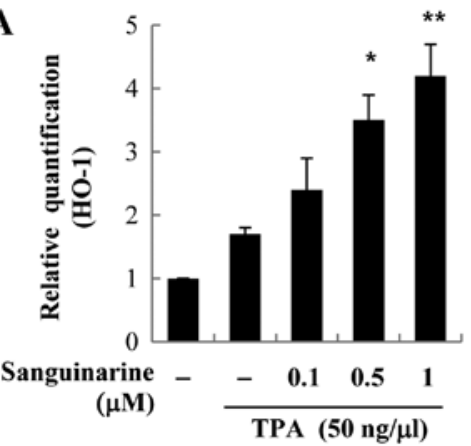

C

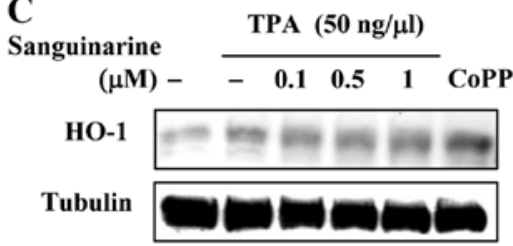

$\mathbf{E}$

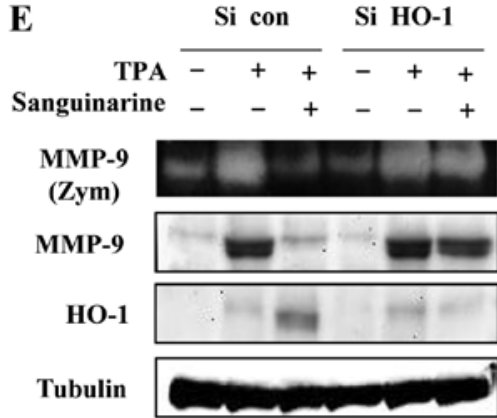

G

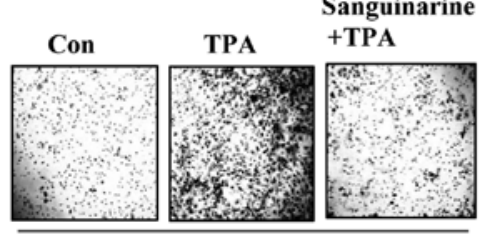

Si con
B

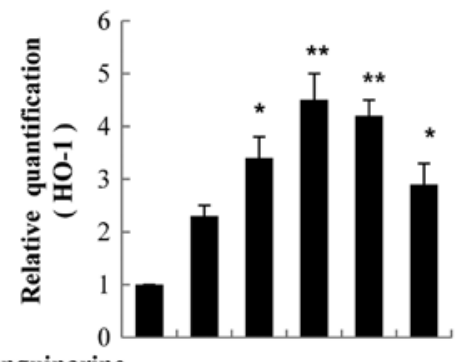

$\begin{array}{lllllll}\text { Sanguinarine } & 0 & 2 & 4 & 8 & 12 & 24(\mathrm{~h})\end{array}$

$(1 \mu \mathrm{M}) \frac{\mathrm{TPA}(50 \mathrm{ng} / \mu \mathrm{l})}{\mathrm{T}}$

D

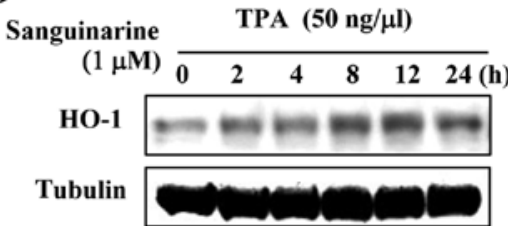

F
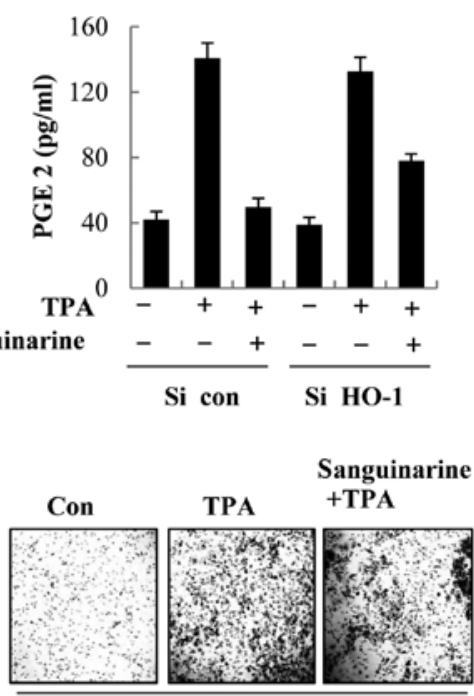

Si HO-1

Figure 5. Effect of HO-1 disruption on the sanguinarine anti-invasive effect in 12- $O$-tetradecanoylphorbol-13-acetate (TPA)-stimulated breast cancer cells (A and B) MCF-7 cells were cultured with increasing concentrations of sanguinarine for $8 \mathrm{~h}$ or with $1 \mu \mathrm{M}$ of sanguinarine for the indicated time-periods. HO-1 mRNA expression was determined by real-time PCR. Each bar represents the mean \pm SE from three independent experiments in each group. " $\mathrm{P}<0.05$; ${ }^{* *} \mathrm{P}<0.01$, both vs. control-treated group. (C and D) Cells were incubated for $12 \mathrm{~h}$ with the indicated concentration of sanguinarine and then incubated with $1 \mu \mathrm{M}$ sanguinarine for the indicated time-periods. Total cellular extracts were prepared, and western blotting was performed. (E and $\mathrm{F}$ ) Cells were transfected with control and HO-1 small interfering RNA (siRNA) using the X-tremeGENE reagent according to the manufacturer's instructions. The cells were treated with $1 \mu \mathrm{M}$ sanguinarine for $1 \mathrm{~h}$ and stimulated with TPA for $24 \mathrm{~h}$, and the activity of MMP-9 and COX-2 was examined by gelatin zymography and PGE enzyme-linked immunosorbent assay (ELISA). (G) Cells were transfected with control and HO-1 siRNA and cells were then treated with sanguinarine (1 $\mu \mathrm{M}$ ), followed by TPA $(50 \mathrm{ng} / \mathrm{ml})$ treatment for $24 \mathrm{~h}$. Cells that invaded the bottom side of the filter were stained with crystal violet and counted under a microscope.

non-toxic concentrations, cell migration and invasion were suppressed. These data implied that the suppressive activity of sanguinarine on cell migration and invasion is not due to its cytotoxic effect. In the present study, we demonstrated that sanguinarine exerts its inhibitory effect on TPA-induced cell migration and invasion in association with the inhibition of MMP-9 expression via the NF- $\mathrm{KB}$ and AP-1 signaling pathways.

The induction of MMP-9 and COX-2 expression is closely associated with tumor angiogenesis, invasion and metastasis, and the suppression of MMP-9 and COX-2 expression plays a crucial role in cancer therapy, since MMPs catalyze the degradation of the ECM and trigger tumor invasion. MMP-9 and COX-2 are overexpressed in aggressive breast cancer and are relevant to the clinical outcome $(5,8,21)$. To further investigate the mechanism of sanguinarine-induced inhibition of cell migration and invasion, we conducted experiments, including gelatin zymography and western blotting, to determine the enzymatic activity and protein levels of MMP-9 and COX-2, respectively. The results showed that the activity and expression of MMP-9 and COX-2 were significantly reduced by treatment with sanguinarine, whereas the expression of MMP-2 and COX-1 were not affected. Accordingly, we suggest that the suppressive effect of sanguinarine on cell migration and invasion is related to the inhibition of enzyme-catalyzed degradation steps in the tumor metastatic process.

Many transcription factors, including NF- $\kappa \mathrm{B}$ and AP-1, are involved in regulating the expression of MMP-9 and COX-2. 
These transcription factors play pivotal roles in metastasis due to their ability to induce the transcription of metastasis-related genes, including MMP-9 and COX-2 (6,7). The results of the present study also revealed that the anti-invasive effects of sanguinarine are associated with the prevention of NF- $\mathrm{\kappa B}$ and AP-1 activation. Sanguinarine inhibits the nuclear translocation and activation of NF- $\mathrm{KB}$ and AP-1. We also investigated the upstream signaling pathways regulating TPA-induced MMP-9 and COX-2 expression and activity. Akt and MAPKs are involved in the expression of MMP-9 and COX-2. We examined whether sanguinarine regulates the activity of MAPKs and found that sanguinarine significantly inhibited Akt and ERK phosphorylation, while having no influence on JNK and $\mathrm{p} 38$. These results suggest that sanguinarine suppresses MMP-9 and COX-2 expression and activity through the inhibition of NF- $\mathrm{KB}, \mathrm{AP}-1, \mathrm{Akt}$ and ERK in breast cancer cells.

Growing evidence has demonstrated that HO-1 exhibits anti-metastatic activity by inhibiting the expression of prometastatic genes, thereby suggesting a potential therapeutic strategy for treating breast cancer metastasis. Several studies have shown an inverse correlation between HO-1 and prometastatic genes $(10,22)$. These results support a role for HO-1 expression as a negative regulator of pro-metastatic genes such as MMP-9 and COX-2. In agreement with these reports, the results of the present study demonstrated that sanguinarine induces the expression of HO-1, providing a potential explanation for its anti-invasive properties. We examined whether HO-1 expression correlates with the inhibition of TPA-induced MMP-9 and COX-2 activities. Knockdown of HO-1 expression using siRNA markedly reversed the inhibitory effects of sanguinarine on MMP-9 and COX-2 activity in TPA-induced breast cancer cells. Moreover, knockdown of endogenous HO-1 in cells suppressed TPA-induced invasion compared to the control siRNA knockdown. These results suggest that the inhibition of breast cancer cell invasion by sanguinarine is consistent with the inhibition of MMP-9 and COX-2 activity via HO-1 expression. Therefore, the anti-invasive effect of sanguinarine might be related to the expression of HO-1 in breast cancer cells.

In conclusion, we demonstrated that sanguinarine inhibits MMP-9 and COX-2 expression and activity in TPA-stimulated MCF-7 breast cancer cells. These effects are not only mediated by the inhibition of the activities of NF- $\mathrm{kB}, \mathrm{AP}-1$, Akt and ERK, but also by the induction of HO-1 expression. This is the first study to demonstrate that sanguinarine suppresses MMP-9 and COX-2 activity by inducing HO-1 expression. The present study provides new insights into the anti-invasive mechanisms of sanguinarine in human breast cancer cells and presents evidence that sanguinarine is a promising candidate for the prevention and treatment of human breast cancer.

\section{Acknowledgements}

The present study was supported by the Basic Science Research Program through the National Research Foundation of Korea (NRF) funded by the Ministry of Education, Science and Technology (2012R1A1A3010601).

\section{References}

1. Love RR and Koroltchouk V: Tamoxifen therapy in breast cancer control worldwide. Bull World Health Organ 71: 795-803, 1993.

2. Agarwal R, Agarwal C, Ichikawa $\mathrm{H}$, et al: Anticancer potential of silymarin: From bench to bed side. Anticancer Res 26: 4457-4498, 2006.

3. Gillard JA, Reed MW, Buttle D, et al: Matrix metalloproteinase activity and immunohistochemical profile of matrix metalloproteinase-2 and -9 and tissue inhibitor of metalloproteinase-1 during human dermal wound healing. Wound Repair Regen 12: 295-304, 2004.

4. Jinga DC, Blidaru A, Condrea I, et al: MMP-9 and MMP-2 gelatinases and TIMP-1 and TIMP-2 inhibitors in breast cancer: correlations with prognostic factors. J Cell Mol Med 10: 499-510, 2006.

5. Kim S, Kim SH, Hur SM, et al: Silibinin prevents TPA-induced MMP-9 expression by down-regulation of COX-2 in human breast cancer cells. J Ethnopharmacol 126: 252-257, 2009.

6. Kim JH, Lee KW, Lee MW et al: Hirsutenone inhibits phorbol ester-induced upregulation of COX-2 and MMP-9 in cultured human mammary epithelial cells: NF- $\kappa \mathrm{B}$ as a potential molecular target. FEBS Lett 580: 385-392, 2006.

7. Anand P, Sundaram C, Jhurani S, et al: Curcumin and cancer: an 'old-age' disease with an 'age-old' solution. Cancer Lett 267: 133-164, 2008.

8. Simeone AM, Nieves-Alicea R, McMurtry VC, et al: Cyclooxygenase-2 uses the protein kinase C/interleukin-8/ urokinase-type plasminogen activator pathway to increase the invasiveness of breast cancer cells. Int J Oncol 30: 785-792, 2007.

9. Otterbein LE and Choi AM: Heme oxygenase: Colors of defense against cellular stress. Am J Physiol Lung Cell Mol Physiol 279: L1029-L1037, 2000.

10. Farombi EO and Surh YJ: Heme oxygenase-1 as a potential therapeutic target for hepatoprotection. J Biochem Mol Biol 39: 479-491, 2006.

11. Jozkowicz A, Was H and Dulak J: Heme oxygenase-1 in tumors: is it a false friend? Antioxid Redox Signal 9: 2099-2117, 2007.

12. Ye F, Feng F and Liu W: Alkaloids from Macleaya cordata. Zhongguo Zhong Yao Za Zhi 34: 1683-1686, 2009 (In Chinese).

13. Pang JX, Ma RQ, Liu LM, et al: Total alkaloid of Macleaya cordata: In vitro cytotoxic effect on Hep3B cells and in vivo antitumor effect in mice. Di Yi Jun Yi Da Xue Xue Bao 25: 325-328, 2005 (In Chinese).

14. Luo XB, Chen B and Yao SZ: Rapid determination of protopine, allocryptopine, sanguinarine and chelerythrine in fruits of Macleaya cordata by microwave-assisted solvent extraction and HPLC-ESI/MS. Phytochem Anal 17: 431-438, 2006.

15. Cornblatt BS, Ye L, Dinkova-Kostova AT, et al: Preclinical and clinical evaluation of sulforaphane for chemoprevention in the breast. Carcinogenesis 28: 1485-1490, 2007.

16. Jang BC, Park JG, Song DK, et al: Sanguinarine induces apoptosis in A549 human lung cancer cells primarily via cellular glutathione depletion. Toxicol In Vitro 23: 281-287, 2009.

17. Kim S, Lee TJ, Leem J, Choi KS, et al: Sanguinarine-induced apoptosis: Generation of ROS, down-regulation of Bcl-2, c-FLIP, and synergy with TRAIL. J Cell Biochem 104: 895-907, 2008.

18. Johnson KD and Bresnick EH: Dissecting long-range transcriptional mechanisms by chromatin immunoprecipitation. Methods 26: 27-36, 2002.

19. Simeone AM, McMurtry V, Nieves-Alicea R, et al: TIMP-2 mediates the anti-invasive effects of the nitric oxide-releasing prodrug JS-K in breast cancer cells. Breast Cancer Res 10: R44, 2008.

20. Formenti S, Felix J, Salonga D, et al: Expression of metastasesassociated genes in cervical cancers resected in the proliferative and secretory phases of the menstrual cycle. Clin Cancer Res 6: 4653-4657, 2000.

21. Larkins TL, Nowell M, Singh S and Sanford GL: Inhibition of cyclooxygenase- 2 decreases breast cancer cell motility, invasion and matrix metalloproteinase expression. BMC Cancer 6: 181, 2006.

22. Hill M, Pereira V, Chauveau C, et al: Heme oxygenase-1 inhibits rat and human breast cancer cell proliferation: mutual cross inhibition with indoleamine 2,3-dioxygenase. FASEB J 19: 1957-1968, 2005. 